\title{
Quantum state reconstruction of the single-photon Fock state
}

\author{
A. I. Lvovsky 1 , H. Hansen, T. Aichele, O. Benson, J. Mlynekt and S. Schiller \\ Fachbereich Physik, Universität Konstanz, D-78457 Konstanz, Germany
}

(October 31, 2018)

We have reconstructed the quantum state of optical pulses containing single photons using the method of phaserandomized pulsed optical homodyne tomography. The single-photon Fock state $|1\rangle$ was prepared using conditional measurements on photon pairs born in the process of parametric down-conversion. A probability distribution of the phase-averaged electric field amplitudes with a strongly nonGaussian shape is obtained with the total detection efficiency of $(55 \pm 1) \%$. The angle-averaged Wigner function reconstructed from this distribution shows a strong dip reaching classically impossible negative values around the origin of the phase space.

a. Introduction States of quantum systems can be completely described by their Wigner functions (WF), the analogues of the classical phase-space probability distributions. Generation of various quantum states and measurements of their WFs is a central goal of many experiments in quantum optics [1-3]. Of particular interest are quantum states whose Wigner function takes on negative values in parts of the phase space. This classically impossible phenomenon is a signature of highly non-classical character of a quantum state.

Quantum states containing a definite number of energy quanta (Fock states $|n\rangle$ ) are paradigmatic in this respect. Their WFs exhibit strong negativities and their marginal distributions are strongly non-Gaussian (Fig. 1). This property reflects the fundamentally non-classical nature of these states as carriers of the particle aspect of light.

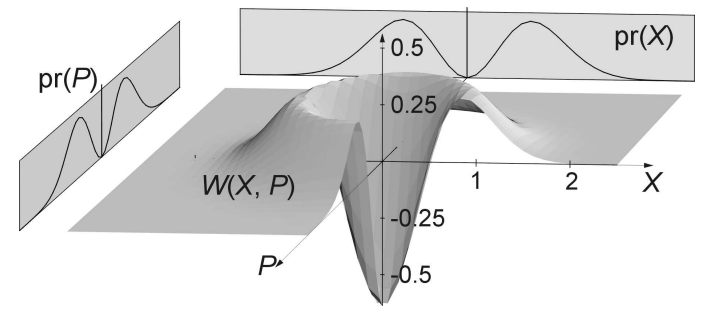

FIG. 1. Theoretical phase space quasiprobability density (Wigner function) of the single-photon state $|1\rangle: \quad W(X, P)=\frac{2}{\pi}\left(4\left(X^{2}+P^{2}\right)-1\right) e^{-2\left(X^{2}+P^{2}\right)}$. $\hat{X}=\left(\hat{a}+\hat{a}^{\dagger}\right) / \sqrt{2}$ and $\hat{P}=\left(\hat{a}-\hat{a}^{\dagger}\right) / \sqrt{2} i$ are normalized non-commuting electric field quadrature observables. Single-quadrature probability densities (marginal distributions) are also displayed.

Generation and complete measurements of the Fock states' WFs were performed on vibrational states of a trapped $\mathrm{Be}^{+}$ion [1]. In the electromagnetic domain, Nogues et al. [2 recently reported the measurement of the WF of a single-photon state in a superconducting microwave cavity at a single point (origin) of the phase space. A full characterization of a Fock state of the electromagnetic field has not been achieved so far.

In this paper we present a measurement of the complete (phase-averaged) Wigner function of the propagating single-photon state $|1\rangle$ in the optical domain. We perform a direct measurement of the dynamical variables of the electromagnetic field, the electric field quadratures, whereby their probability distributions are obtained. The Wigner function is then reconstructed from the measured distributions. This method, homodyne tomography, has been established as a reliable technique of reconstructing quantum states in the optical domain. Previously, it has been applied to classical and weakly non-classical states of the light field, such as vacuum, coherent, thermal and squeezed states, in the continuous-wave as well as in the pulsed regime 3 .

The main challenge associated with a tomographic characterization of the single-photon state is the preparation of this state in a well-defined spatio-temporal mode. We solve this task by employing conditional state preparation on a photon pair born in the process of parametric down-conversion [4,5]. The two generated photons are separated into two emission channels according to their propagation direction (Fig. 2). A single-photon counter is placed into one of the emission channels (labeled trigger) to detect photon pair creation events and to trigger the readout of a homodyne detector placed in the other (signal) channel [6].

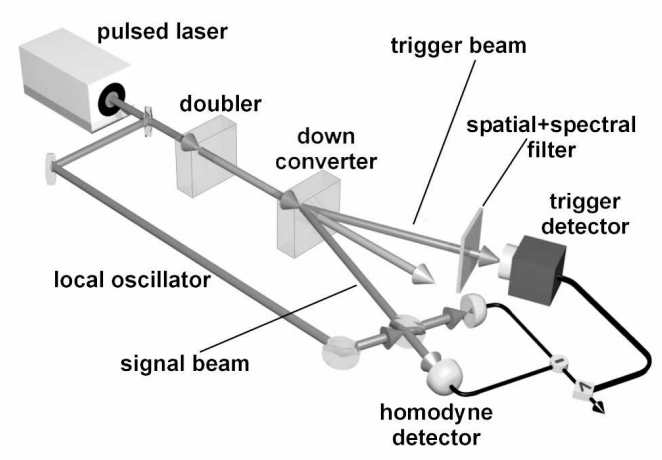

FIG. 2. Simplified scheme of the experimental setup

b. Theory The process of pulsed 2-photon downconversion produces strongly correlated photon pairs. 
The generated biphoton state can be written as

$$
|\Psi\rangle=N\left(|0,0\rangle+\int d \vec{k}_{s} d \vec{k}_{t} \Phi\left(\vec{k}_{s}, \vec{k}_{t}\right)\left|1_{\vec{k}_{s}}, 1_{\vec{k}_{t}}\right\rangle\right),
$$

where $N$ is a normalization constant, $\vec{k}_{s}$ and $\vec{k}_{t}$ denote the signal and trigger beam wave vectors, respectively, and the function $\Phi\left(\vec{k}_{s}, \vec{k}_{t}\right)$ carries the information about the amplitude as well as the transverse and longitudinal structure of the photon pair generated [8].

A single-photon Fock state is prepared from $|\Psi\rangle$ by projecting this state onto a photon count event in the trigger beam path:

$$
\hat{\rho}_{s}=\operatorname{Tr}_{t}|\Psi\rangle\langle\Psi| \hat{\rho}_{t},
$$

where $\hat{\rho}_{t}$ denotes the state ensemble selected by the trigger and the trace is taken over the trigger states.

The trigger state ensemble $\hat{\rho}_{t}$ is determined by the spatial and spectral filtering in the trigger channel:

$$
\hat{\rho}_{t}=\int d \vec{k}_{t} T\left(\vec{k}_{t}\right)\left|1_{\vec{k}_{t}}\right\rangle\left\langle 1_{\vec{k}_{t}}\right|,
$$

where $T\left(\vec{k}_{t}\right)$ is the spatiotemporal transmission function of the filter. Note that although $|\Psi\rangle$ represents a pure state, $\hat{\rho}_{t}$ and hence $\hat{\rho}_{s}$ are statistical mixtures. However, if sufficiently tight filtering is applied to the trigger channel (so that $T\left(\vec{k}_{t}\right)$ is much narrower than the spatial and spectral width of the pump beam), $\hat{\rho}_{s}$ will approach a pure single-photon state [7]. In this case, the signal photons are prepared in a relatively well-defined optical mode suitable for homodyne detection.

It is important to understand that the "signal beam" as shown in Fig. 2 is not an optical beam in the traditional sense. The down-converted photons are in fact emitted randomly over a wide solid angle. The optical mode of the signal state is created non-locally only when a photon of a pair hits the trigger detector and is registered. The coherence properties of this mode are determined by the optical mode of the pump and the spatial and spectral filtering in the trigger channel.

Once the approximation $\hat{\rho}_{s}$ of the Fock state is prepared, it is subjected to balanced homodyne detection. The signal wave is overlapped on a beamsplitter with a relatively strong local oscillator (LO) wave in the matching optical mode. The two fields emerging from the beamsplitter are incident on two high-efficiency photodiodes whose output photocurrents are subtracted. The photocurrent difference is proportional to the value of the electric field operator $\hat{E}(\theta)$ in the signal mode, $\theta$ being the relative optical phase of the signal and LO.

For each phase $\theta$ one measures a large number $N$ of samples of $\hat{E}(\theta) \propto \hat{X}_{\theta} \equiv \hat{X} \cos \theta+\hat{P} \sin \theta$, so that their histogram (i. e. the marginal distribution) $\operatorname{pr}\left(X_{\theta}\right)$ can be determined. The latter is related to the WF as follows:

$$
\begin{aligned}
\operatorname{pr}\left(X_{\theta}\right) & =\left\langle X_{\theta}\left|\hat{\rho}_{\text {meas }}\right| X_{\theta}\right\rangle \\
& =\int_{-\infty}^{\infty} W(X \cos \theta-P \sin \theta, X \sin \theta+P \cos \theta) d P
\end{aligned}
$$

where $\hat{\rho}_{\text {meas }}$ is the density matrix of the state being measured. The marginal distribution $\operatorname{pr}\left(X_{\theta}\right)$ can be envisioned as a density projection of the WF $W(X, P)$ onto a vertical plane oriented at an angle $\theta$ with respect to the plane $P=0$ (Fig. 1). From the set of marginal distributions $\operatorname{pr}\left(X_{\theta}\right)$ for a large number of phase angles $\theta$ the WF of $\hat{\rho}_{\text {meas }}$ can be reconstructed via a procedure similar to the one used in medical computer tomography [9].

In a perfect experiment, $\hat{\rho}_{\text {meas }}=|1\rangle\langle 1|$, where the single-photon state is in the optical mode which matches that of the local oscillator. In reality, various imperfections (such as optical losses in the signal arm, inefficient photodiodes, dark counts, non-ideal matching between the signal and the LO optical modes 10) cause an admixture of the vacuum $|0\rangle$ to the measured state, so that

$$
\hat{\rho}_{\text {meas }}=\eta|1\rangle\langle 1|+(1-\eta)| 0\rangle\langle 0|,
$$

$\eta$ being the measurement efficiency. It is remarkable that all these effects act upon $\hat{\rho}_{\text {meas }}$ in a similar way, so that their effect can be expressed in a single number $\eta$ which is a product of efficiencies associated with individual parts of the setup. The value of $\eta$ is crucial for this experiment as it strongly influences the shape of the measured marginal distributions and the reconstructed Wigner function (Fig. 3) [6].

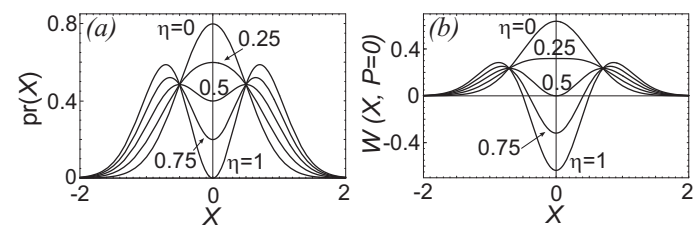

FIG. 3. Effect of the non-perfect measurement efficiency $\eta$ on the marginal distribution (a) and the reconstructed WF (b). For the WF, cross-sections by the plane $P=0$ are shown. Negative values require $\eta>0.5$.

In our experiment we used a simplified scheme in which the phase $\theta$ varied randomly, so that we only measured a single phase-randomized marginal distribution $\operatorname{pr}_{\mathrm{av}}(X)=\left\langle\operatorname{pr}\left(X_{\theta}\right)\right\rangle_{\theta}$. This does not change the measurement result for quantum states with rotationally symmetric Wigner functions such as those described by Eq. (5). The phase-averaged WF $W(R)$ is obtained from $\operatorname{pr}_{\mathrm{av}}(X)$ via the Abel transformation 9, 11]:

$$
W(R)=-\frac{1}{\pi} \int_{R}^{\infty} \frac{d \mathrm{pr}_{\mathrm{av}}(X)}{d X}\left(X^{2}-R^{2}\right)^{-1 / 2} d X .
$$

From the phase-randomized marginal distributions one can also directly infer diagonal elements $\rho_{n n}$ of the state density matrix in the Fock basis,

$$
\rho_{n n}=\pi \int_{-\infty}^{\infty} \operatorname{pr}_{\mathrm{av}}(X) f_{n n}(X) d X,
$$

where $f_{n n}(X)$ are the amplitude pattern functions 13. which are independent of the optical state being sampled. The statistical uncertainty of the reconstructed $\rho_{n n}$ is 


$$
\left\langle\sigma_{n n}^{2}\right\rangle=\frac{1}{N} \int_{-\infty}^{\infty} \operatorname{pr}_{\mathrm{av}}(X)\left(\pi f_{n n}(X)\right)^{2} d X
$$

where $N$ is the total number of field samples acquired.

c. Experimental Setup We employed a mode-locked Ti:sapphire laser (Spectra Physics Tsunami) in combination with a pulse picker to obtain transform-limited pulses at $790 \mathrm{~nm}$ with a repetition rate $816 \mathrm{kHz}$ and a pulse width of $1.6 \mathrm{ps}$. Most of the radiation was frequency doubled in a single pass through a 3 -mm LBO crystal yielding $100 \mu \mathrm{W}$ at $395 \mathrm{~nm}$ and then passed on to a 3-mm BBO crystal for down-conversion.

Down-conversion occurred in a type-I frequencydegenerate, but spatially non-degenerate configuration, with 790 -nm photon pairs emitted at angles $\pm 6.8^{\circ}$ with respect to the pump beam. The BBO crystal was cut at $\theta=35.7^{\circ}, \phi=0^{\circ}$, so that the direction of the walk-off of the $395 \mathrm{~nm}$ pump beam inside the crystal coincided with the direction of the signal beam so as to minimize distortions of the signal spatial mode ("hot spot" downconversion, [12]). The short crystal lengths allowed us to avoid group-velocity mismatch effects which would have complicated temporal mode matching to the LO pulse.

The trigger photons passed through a spatial filter and a 0.3 -nm interference filter centered at the laser wavelength. They were then detected by an EG\&G SPCMAQ-131 single-photon detector (quantum efficiency $60 \%$, dark count rate $<15 \mathrm{~s}^{-1}$ ) at a rate of about $0.25 \mathrm{~s}^{-1}$. Such a low pair production rate made the effect of Fock states with $n>1$ negligible. Precise (within $0.6 \mathrm{~ns}$ ) gating of the count events with the laser pulses allowed us to eliminate most of the dark counts, thereby reducing their contribution to about $2 \%$ of all trigger events.

We used a small fraction of the original optical pulses from the pulse picker - split off before the frequencydoubler - as the local oscillator for the homodyne system. Achieving a good spatial and temporal mode matching between the LO and the photons in the signal channel constituted a major challenge in this experiment due to extremely low intensity of the field in the signal mode. To this end, a fraction of the laser output power was directed into the $\mathrm{BBO}$ crystal from the back along the trigger beam path so that it passed through the spatial filter in the trigger channel. Inside the crystal these alignment pulses were temporally and spatially overlapped with the pump to produce a difference frequency (DFG) emission into an optical mode which modeled, to a good precision, that of the conditionally prepared signal photons. This mode was then matched to that of the local oscillator by observing an interference pattern between the two beams. A visibility on the level of $v=83 \pm 1 \%$ was reached.

The method of conditional state preparation also established special requirements for the homodyne detector electronics. The detector needed to resolve quantum shot noises of individual laser pulses at a $0.8-\mathrm{MHz}$ repetition rate. Details on design and performance of the homodyne system developed will be published elsewhere [14].
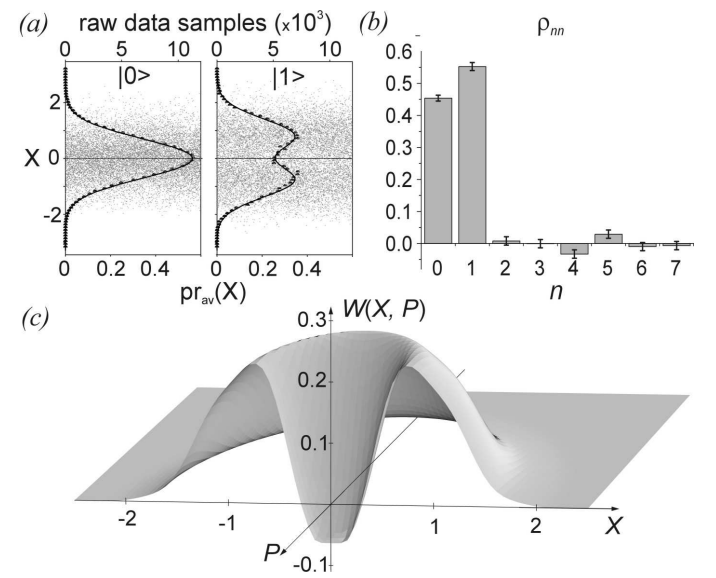

FIG. 4. Experimental results: a) raw quantum noise data for the vacuum (left) and Fock (right) states along with their histograms corresponding to the phase-randomized marginal distributions; b) diagonal elements of the density matrix of the state measured. c) reconstructed WF which is negative near the origin point. The measurement efficiency is $55 \%$.

d. Results and discussion In a 14-hour experimental run about 200,000 vacuum state and 12,000 Fock state samples were acquired (Fig. 4a). Both data sets were then binned up to obtain their statistical distributions. A Gaussian distribution was fit to the vacuum state noise spectrum by varying its X-scale and point of origin.

The best fit parameters of the vacuum state were used to scale the Fock state data. The latter was then fit by the theoretical marginal distribution of the ensemble (5) to find the measurement efficiency $\eta$. The best fit efficiency value was $\eta=0.55$. Using the Abel transform (6) the phase-randomized WF of the observed quantum state was reconstructed (Fig. 4c). As expected, it exhibits negativity around the origin point, with a minimum value $W(0,0)=-0.062$.

The diagonal elements of the density matrix have been evaluated along with their statistical errors by applying the quantum state sampling method as defined by Eqs. (7), 8) directly to the rescaled raw data. We found $\rho_{11}=0.553 \pm 0.013$ in agreement with the value of $\eta$ obtained by fitting the marginal distribution (Fig. 4b). Substituting this quantity into Eq. (5) and calculating the corresponding value of the WF we find $W(0,0)=$ $-0.067 \pm 0.016$, in agreement with the above value determined from the marginal distribution. The uncertainty of \pm 0.016 gives an estimation for the accuracy to which the negative value of the Wigner function has been determined in this experiment.

The values of $\rho_{00}$ and $\rho_{11}$ for the vacuum state were found to be $0.9975 \pm 0.0029$ and $0.0021 \pm 0.0032$, respectively. These quantities being equal to their ideal values within the statistical errors indicates that no unknown conditions (e.g. mechanical vibrations) are present that might bring the apparent measurement efficiency above its actual value. The systematic experimental errors (e.g. technical noise in the homodyne detector) were insignif- 
icant and would result in decrease of the measured efficiency. It is thus impossible that neglecting these errors could bring about a too optimistic estimate of $\eta$.

What are the main factors reducing the measurement efficiency? The non-perfect match between the optical modes of LO and DFG fields diminishes the efficiency by a factor of $v^{2}=0.69 \pm 0.02$. The fact that the DFG wave does not perfectly mimic the conditionally prepared mode of the single photon causes an additional reduction by $\times 0.95$. A factor of 0.90 arises from losses in the signal beam path and non-perfect quantum efficiency of the photodiodes in the homodyne detector. Finally, a $2 \%$ reduction occurs due to false trigger count events. Combining all the above factors we obtain the upper limit estimate of the quantum efficiency as $(57 \pm 2) \%$, which is in good agreement with the experimental value of $55.3 \%$.

e. Conclusion and outlook We have reconstructed the phase-averaged Wigner function and the density matrix diagonal elements of an optical single-photon Fock state |1) with a total measurement efficiency of $55.3 \pm 1.3 \%$ using the method of phase-randomized pulsed optical homodyne tomography. The reconstructed WF is of nonGaussian shape and exhibits negative values around the origin of phase space, reflecting the strongly non-classical character of the state $|1\rangle$ as a particle state of the light field. Single-photon Fock states were prepared in a welldefined electromagnetic mode by conditional measurements on photon pairs created in the process of parametric fluorescence. The measurement technique and error analysis were checked by performing a simultaneous measurement on the vacuum state. Major experimental inefficiency factors have been identified and quantified. This experiment represents the first quantum tomography measurement of a highly nonclassical state of the electromagnetic field.

Relatively straightforward modifications of the setup would allow tomography measurements of displaced Fock states [15] and 1-photon added coherent states [16]. Of special interest is the entangled state $|0,1\rangle-|1,0\rangle$ generated when a single photon is incident on a beamsplitter. This state can also be used to demonstrate nonlocality of a single photon [17]. In a more distant future, arbitrary quantum states of the light field might be generated via repeated down-conversion 18].

We thank S. Eggert, C. Hettich and P. Lodahl for their help in building up the experimental setup. A. L. is supported by the Alexander von Humboldt Foundation. This work was funded by the Deutsche Forschungsgemeinschaft.

* email: Alex.Lvovsky@uni-konstanz.de

$\dagger$ Present address: President, Humboldt-Universität zu Berlin, D-10099 Berlin, Germany
¥ Present address: Institut für Experimentalphysik, Heinrich-Heine-Universität Düsseldorf, D-40225 Düsseldorf, Germany

[1] D. Leibfried et al. Phys. Rev. Lett. 77, 4281 (1996)

[2] G. Nogues et al. Phys. Rev. A 62, 054101 (2000).

[3] D. T. Smithey et al. Phys. Rev. Lett. 70, 1244 (1993); G. Breitenbach, S. Schiller, and J. Mlynek, Nature 387, 471 (1997); M. Vasilyev et al., Phys. Rev. Lett. 84, 2354 (2000)

[4] C. K. Hong and L. Mandel, Phys. Rev. Lett. 56, 58 (1986)

[5] P. Grangier, G. Roger and A. Aspect, Europhys. Lett. 1, 173 (1986)

[6] B. Yurke and D. Stoler, Phys. Rev. A 36, 1955 (1987)

[7] Z. Y. Ou, Qu. Semiclass. Opt. 9, 599 (1997)

[8] A. Joobeur et al., Phys. Rev. A 53, 4360 (1996)

[9] for details on quantum tomography and inverse Radon transformation, see U. Leonhardt, Measuring the quantum state of light, Cambridge University Press, 1997

[10] F. Grosshans and P. Grangier, Eur. Phys. J. D 14, 119 (2001)

[11] U. Leonhardt and I. Jex, Phys. Rev. A 49, 1555 (1994)

[12] K. Koch et al., IEEE J. Quant. El. 31, 769 (1995)

[13] G. M. D'Ariano, U. Leonhardt, and H. Paul, Phys. Rev. A 52, R1801 (1995); U. Leonhardt and M. G. Raymer, Phys. Rev. Lett. 76, 1985 (1996)

[14] H. Hansen et al. quant-ph/0104084

[15] K. E. Cahill and R. J. Glauber, Phys. Rev. 177, 1857 (1969)

[16] G. S. Agarwal and K. Tara, Phys. Rev. A 43, 492 (1990); C. T. Lee, Phys. Rev. A 52, 3374 (1995)

[17] S. M. Tan, D. F. Walls, and M. J. Collett, Phys. Rev. Lett. 66, 252 (1991); K. Banaszek and K. Wodkiewicz, ibid. 82, 2009 (1999); K. Jacobs and P. L. Knight, Phys. Rev. A 54, 3738 (1996)

[18] J. Clausen et al., quant-ph/0007050 